\title{
Expression and significance of PTEN and miR-92 in hepatocellular carcinoma
}

\author{
BIN ZHAO $^{1 *}$, YUETING ZHU $^{2 *}, \mathrm{KAI} \mathrm{CUI}^{3}, \mathrm{JIE} \mathrm{GAO}^{4}, \mathrm{FACHANG} \mathrm{YU}^{4}$, LIANG CHEN $^{5}$ and SHENG LI $^{6}$ \\ ${ }^{1}$ School Hospital, Shandong University; ${ }^{2}$ Hospital for Reproductive Medicine Affiliated to Shandong University; \\ ${ }^{3}$ Key Laboratory for Rare and Uncommon Diseases of Shandong Province; ${ }^{4}$ Shandong Academy of Medical Sciences; \\ ${ }^{5}$ Shandong Provincial Hospital; ${ }^{6}$ Department of Hepatobiliary Surgery, Shandong Cancer Hospital, \\ Jinan, Shandong 250011, P.R. China
}

Received October 23, 2012; Accepted January 28, 2013

DOI: $10.3892 / \mathrm{mmr} .2013 .1406$

\begin{abstract}
The aim of this study was to investigate the correlation between the expression levels of the tumor suppressor gene phosphatase and tensin homolog (PTEN) and the microRNA (miRNA) miR-92 in hepatocellular carcinoma (HCC) and paracancerous tissue. Immunohistochemistry [streptavidin-peroxidase (SP)] and quantitative reverse transcriptase-polymerase chain reaction (qRT-PCR) were used to detect the expression of PTEN and miR-92 in 15 cases of HCC and the corresponding paracancerous tissues. The correlation between PTEN and miR-92 was analyzed. The expression of PTEN mRNA and protein was significantly lower in HCC compared with that in paracancerous tissues $(\mathrm{P}<0.05)$. miR-92 showed the opposite expression pattern $(\mathrm{P}<0.05)$. Additionally, the mRNA levels of PTEN and miR-92 showed a significantly negative correlation with each other $(\mathrm{r}=-0.858, \mathrm{P}<0.05)$. In conclusion, PTEN and miR-92 have different roles in the development of HCC. The combined detection of PTEN and miR-92 may provide critical clinical evidence for the early diagnosis and prognosis of HCC.
\end{abstract}

\section{Introduction}

Hepatocellular carcinoma (HCC) accounts for $\sim 80-90 \%$ of the primary liver cancer cases worldwide. Additionally, it is the third most common cause of cancer-related mortality after gastric and esophageal cancer (1). Surgery is considered to be the primary treatment option. Without liver transplantation, the 5 -year survival rate of patients with $\mathrm{HCC}$ is $<5 \%$. Therefore, research focused on $\mathrm{HCC}$ is critical, and this disease has

Correspondence to: Dr Sheng Li, Department of Hepatobiliary Surgery, Shandong Cancer Hospital, No. 440 Jiyan Road, Jinan, Shandong 250011, P.R. China

E-mail: drlisheng@sohu.com

${ }^{*}$ Contributed equally

Key words: hepatocellular cancer, phosphatase and tensin homolog, miR-92 attracted considerable attention from international medical groups (2). Tumor development in HCC is a complex process involving multiple factors and stages, while the detailed mechanism of HCC tumor development has not yet been fully elucidated. However, cell proliferation and apoptosis are the primary drivers of HCC tumorigenesis, as in most types of cancer. The tumor suppressor phosphatase and tensin homolog (PTEN) and the newly discovered hepatocellular biomarker miR-92 represent the apoptosis-related factors that may be important in HCC development.

The PTEN gene is located on chromosome 10q23.3. Its protein product is a tumor suppressor with a dual phosphatase activity. It dephosphorylates the D3 position on phosphatidylinositol 3,4,5-trisphosphate (PIP3) to negatively regulate the phosphatidylinositol 3-kinase (PI3K)/Akt signaling pathway. This antagonizes the growth and survival signals elicited by the PI3K pathway and induces cell death and cell cycle arrest. Thus, PTEN activity inhibits tumor development (3). The mutation and inactivation of tumor suppressor genes, such as PTEN, leads to uncontrolled cell proliferation and survival, which subsequently increases the likelihood of tumor formation. Moreover, PTEN mutation is potentially associated with the histological grading of HCC tumors and the invasive metastatic phenotype of the disease. PTEN mutations have been identified in a number of other types of cancer, including gastric, hepatic, breast, endometrial and prostate cancer $(4,5)$. In these types of tumors, loss of PTEN function is critical for tumorigenesis since it has been shown to regulate the cell cycle, apoptosis, invasion and metastasis of tumor cells (6).

MicroRNAs (miRNAs) are a class of non-coding single-stranded RNAs (20-24 nucleotides), which are encoded by the genomes of higher eukaryotes. Mature miRNAs are formed through a series of processing steps in the nucleus and cytoplasm (7). miRNAs exert their regulatory effects by binding to the 3 ' untranslated regions (UTRs) of target gene mRNAs. This results in degradation of the target mRNA by the RNA-induced silencing complex (RISC) or by inhibition of the target mRNA translation (8). Previous studies have demonstrated that the expression levels of various miRNAs differ significantly in different tissues and at different developmental stages. Notably, each miRNA may have multiple target genes, and multiple miRNAs may regulate a single gene. The 
microRNA-17-92 (miR-17-92) gene family is considered to play a critical role in the normal development of the lung, heart and immune system. This family may also be significant in tumor formation, since several miRNA family members have been shown to be upregulated in multiple tumor types. miR-92 is a member of the miR-17-92 family and has been previously described to be a proto-oncogene. In fact, it has been shown to increase proliferation and inhibit apoptosis, thus leading to tumorigenesis (9). Mice with specific expression of the miR-17-92 gene family in lymphocytes exhibited an increased proliferation of this cell type, developed autoimmune disease, and died prematurely. In these mice, miR-17-92 promoted the proliferation of lymphocytes and attenuated apoptosis by inhibiting PTEN and increasing Bim expression (10). Since miR-92 plays a specific role in apoptosis, PTEN may also be critical in HCC. Therefore, both may have a negative correlation in HCC. In this study, we used immunohistochemistry and quantitative reverse transcription-polymerase chain reaction (qRT-PCR) to investigate the expression of miR-92 and PTEN as well as the correlation between the two. Their influence on HCC tumor development was examined and our data were related to useful clinical parameters, such as diagnosis and prevention.

\section{Materials and methods}

Subjects. A total of 15 patients who underwent surgery at the Department of General Surgery, Qilu Hospital of Shandong University (Jinan, China) between March 2008 and April 2009 with integrated medical records were included in this study. The study was approved by the ethics committee of Shandong University. Resected tissue diagnosis for HCC was performed using postoperative pathological methods. The patients did not receive any hormonal therapy prior to surgery, and did not suffer from any complicating diseases in the nervous or endocrine systems. The differentiation status of each tumor was defined according to the Edmondson grading system, in which the HCC is defined as grade I, II, III or IV. Grades I and II are considered to be highly differentiated, while grades III and IV are considered to be less differentiated. Fresh specimens of the $\mathrm{HCC}$ and paracancerous tissues $(2 \mathrm{~cm}$ in distance from the edge of the tumor) were obtained by resection, fixed in $4 \%$ formalin and prepared into paraffin sections. The remaining tissues were preserved in a $-80^{\circ} \mathrm{C}$ freezer after freezing in liquid nitrogen.

Reagents. The following reagents were used: rabbit anti-human PTEN monoclonal antibody (Cell Signaling Technology, Inc., Beverly, MA. USA); streptomycin avidin-peroxidase kit and concentrated DAB kit (Beijing Zhongshan Goldenbridge Biotechnology, Co., Ltd., Beijing, China); diethyl pyrocarbonate (DEPC; Takara Biotechnology, Dalian, China); TRIzol, SYBR fluorescent real-time PCR kit and reverse transcription kit (Invitrogen, Carlsbad, CA, USA); TaqMan ${ }^{\circledR}$ MicroRNA kit (Applied Biosystems, Foster City, CA, USA); and primers for PTEN and $\beta$-actin (BGI Life Technologies, Beijing, China).

\section{Methods}

PTEN and miR-92 expression in HCC and paracancerous tissues using streptavidin-peroxidase (SP) methods. Experimental procedures were performed according to the manufacturer's instructions for two-step detection.
Positive results were defined as yellow-brown staining in the cytoplasm of hepatocytes. Under a light microscope (magnification, x400), 10 different non-overlapping visual fields were randomly chosen in each specimen. Stained cells were counted by artificial counts, and the mean values were used for comparisons among the groups. The positive cells were divided into 4 different grades based on their number and intensity: cells with no obvious difference in staining intensity and background were defined as (-); numbers of positive cells $<10 \%$ with mostly weak staining intensity were defined as (+); numbers of positive cells $>50 \%$ with mild to strong staining intensity were defined as $(+++)$; numbers of positive cells and staining intensity between $(+)$ and $(+++)$ were considered $(++)$. The criteria used to assess the immunohistochemical results have been previously described (11).

PTEN and miR-92 expression in HCC and paracancerous tissues as detected using qRT-PCR

Extraction of total RNA from carcinoma and paracancerous tissues. Tissues $(100 \mu \mathrm{g})$ were harvested and pulverized into powder. TRIzol $(1 \mathrm{ml})$ was added to mix evenly, and the powder was placed into a 1.5-ml Eppendorf tube. Chloroform $(0.2 \mathrm{ml})$ was added and the tube was vigorously mixed for $15 \mathrm{sec}$. The tube was then placed at room temperature for $2 \mathrm{~min}$ and centrifuged at $12,000 \mathrm{x}$ g for $30 \mathrm{~min}$. The upper aqueous phase was obtained and placed into a new EP tube. The same proportion of isopropyl alcohol was added. The EP tube was inverted 10 times, and then centrifuged at $12,000 \times \mathrm{g}, 4^{\circ} \mathrm{C}$ for $30 \mathrm{~min}$. Supernatants were discarded, ethanol was added and mixed evenly, and the solution was centrifuged at $12,000 \mathrm{x} \mathrm{g}$ $4^{\circ} \mathrm{C}$, for $10 \mathrm{~min}$. The supernatants were again discarded, and the remaining portion was dried at room temperature for $10 \mathrm{~min}$. The extracted RNA was dissolved in $30 \mu \mathrm{l}$ DEPC water. The purity and density of RNA were determined using UV spectrophotometry and 1\% agarose gel electrophoresis was used to detect the integrity of the RNA. Finally, the RNA was preserved in a $-80^{\circ} \mathrm{C}$ freezer.

Reverse transcription of synthetic $c D N A$ and $q R T-P C R$. The TaqMan ${ }^{\circledR}$ MicroRNA kit was used. U6 snRNA was used as an internal control to perform qRT-PCR for the detection of the differential expression of miR-92. The primer used for miR-92 was obtained from Applied Biosystems. The reaction system and conditions used were as described in the manufacturer's instructions. Three replicates were used for each detection index, and the formula $2^{-\Delta \Delta \mathrm{CT}}$ was used for the analysis of the final data. cDNA synthesis was performed by collecting equal amounts of total RNA, followed by the application of reverse transcription when qRT-PCR was performed. A reaction volume of $50 \mu \mathrm{l}$ was used for each gene amplification. Each reaction mixture consisted of $2 \mu \mathrm{l}$ PCR products, $5 \mu 110 \mathrm{X}$

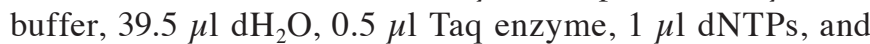
$10 \mu \mathrm{l}$ primers for PTEN and control $\beta$-actin. The primers used were the following: PTEN: upstream, 5'-TCCACAAA CAGAACAAGATG-3' and downstream, 5'-CTGGTCCTGG TATGAAGAAT-3'; $\beta$-actin: upstream, 5'-CTAAGTCATAG TTCCGCCTAGAAGCA-3' and downstream, 5'-TGGCACC CAGCACAATGAA-3'. The qRT-PCR conditions for PTEN and $\beta$-actin were: $95^{\circ} \mathrm{C}$ for $10 \mathrm{~min}, 95^{\circ} \mathrm{C}$ for $10 \mathrm{sec}$, and then $60^{\circ} \mathrm{C}$ for $60 \mathrm{sec}$ for 24 repeated cycles. 

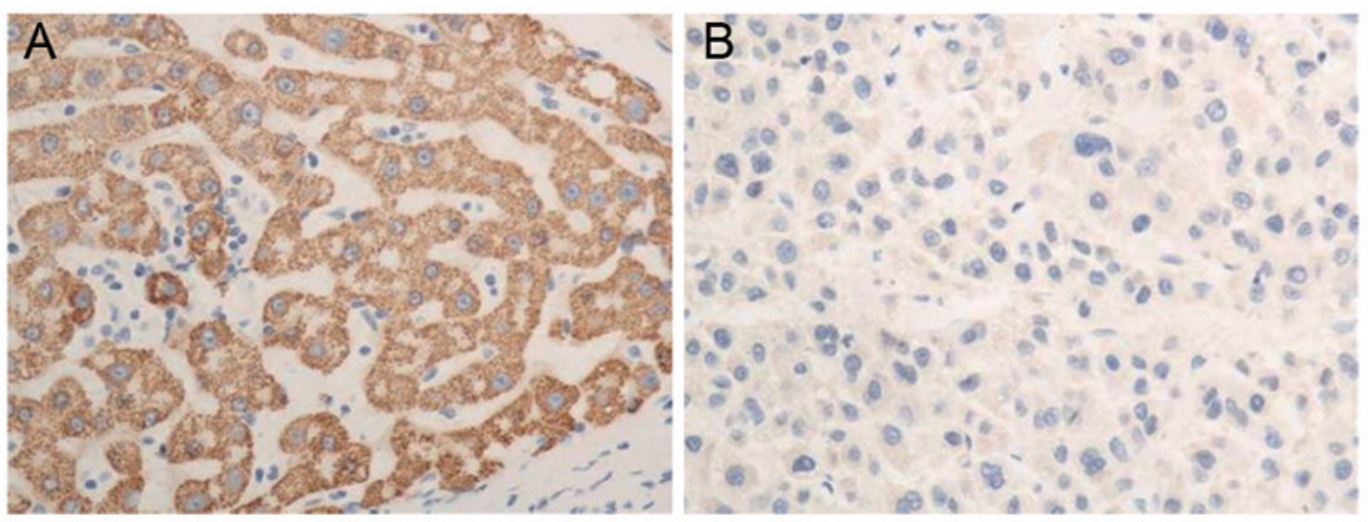

Figure 1. PTEN protein expression in different tissues as detected by immunocytochemistry (DAB; magnification, $\mathrm{x} 400)$. (A) Paracancerous and (B) cancerous tissue. The mean counts of PTEN-positive cells in the cancerous and paracancerous tissue were calculated $(\mathrm{P}<0.05)$. PTEN, phosphatase and tensin homolog.

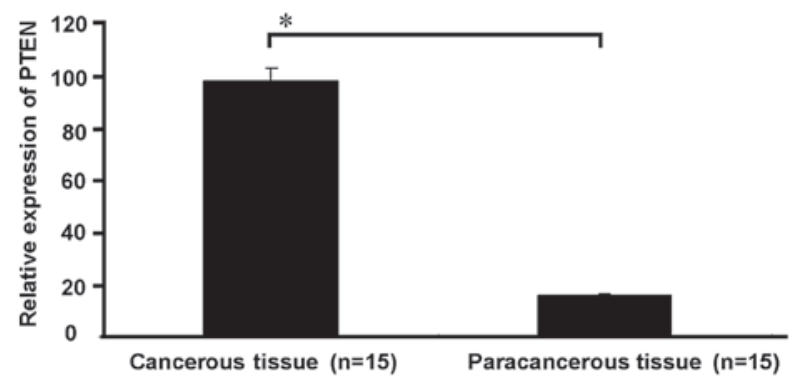

Figure 2. Comparison of relative expression of PTEN between cancerous and paracancerous tissue. ${ }^{*} \mathrm{P}<0.05$. PTEN, phosphatase and tensin homolog.

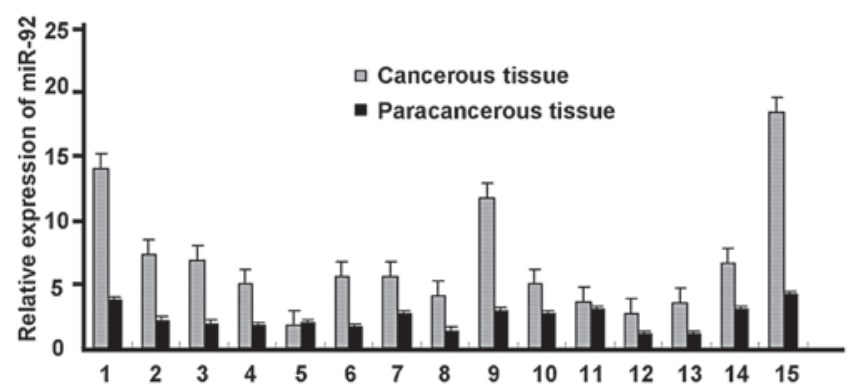

Figure 3. Comparison of relative expression of miR-92 between cancerous and paracancerous tissue $(\mathrm{P}<0.05)$.

Statistical analysis. SPSS 16.0 software (SPSS, Inc., Chicago, IL, USA) was used to conduct the analysis, and the Student's t-test was used for comparison between groups. ANOVA was used for comparisons among groups. Pearson's correlation analysis was used to analyze the correlation of the two indices. $\mathrm{P}<0.05$ was considered to indicate a statistically significant difference.

\section{Results}

Expression of PTEN protein and PTEN $m$ RNA in the HCC and paracancerous tissues. PTEN mRNA was detected as a clear band at $517 \mathrm{bp}$ in the 15 cases of HCC and normal hepatic tissues. A band at 712 bp was detected for $\beta$-actin, which served as an internal control.
Table I. Correlation analysis between miR-92 and PTEN in hepatocellular carcinoma tissues $(\mathrm{r}=-0.858, \mathrm{P}<0.05$; $\mathrm{n}=15)$.

\begin{tabular}{lcc}
\hline & PTEN, $\mathrm{n}$ & miR-92, $\mathrm{n}$ \\
\hline High expression & 2 & 14 \\
Low expression & 13 & 1 \\
\hline
\end{tabular}

PTEN, phosphatase and tensin homolog.

The immunohistochemical results demonstrated positive staining for PTEN protein in the nuclei of tumor cells, and yellow-brown particles were generated. The expression level of PTEN protein in each HCC tissue sample was significantly lower compared with that in the corresponding paracancerous tissue $(\mathrm{P}<0.05$, Fig. 1$)$. The qRT-PCR results indicated that the PTEN mRNA expression level in $13(86.7 \%)$ of the cases of HCC was significantly lower compared with the PTEN mRNA expression level in the corresponding paracancerous tissue. The expression level of PTEN showed a significant difference between the HCC and paracancerous tissues ( $\mathrm{P}<0.05$, Fig. 2).

Expression of miR-92 mRNA in HCC and paracancerous tissues. The miR-92 mRNA expression level was significantly higher in $14(93.3 \%)$ of the cases of HCC than in the corresponding paracancerous tissue. However, in one case there was no significant difference in the miR-92 expression level between the HCC and paracancerous tissues (Fig. 3).

Correlation of PTEN and miR-92 expression levels in HCC. The relative amounts of miR-92 expression were gradually increased with the degree of malignancy in HCC, while the relative amounts of PTEN gene expression gradually decreased. Pearson's correlation analysis indicated that the expression of miR-92 showed a significant negative correlation with PTEN expression in HCC ( $\mathrm{r}=-0.858, \mathrm{P}<0.05$, Table I).

\section{Discussion}

Tumorigenesis is a complex process influenced by various genes and involving various stages. Studies have demonstrated 
that the abnormal expression of multiple genes is implicated in the development of HCC. Proliferation and apoptosis are known to be critical for tumor formation and growth (12). The development of HCC is associated with high malignancy, poor therapies and gene expression changes. The PTEN gene is a tumor suppressor gene with dual-specific phosphatase activity, which is a critical signaling molecule in the regulation of cell proliferation and apoptosis, thus inhibiting tumorigenesis. The PTEN gene regulates normal physiology mainly through the PIP3, FAK and MAPK signaling pathways (13). A study demonstrated that multiple human tumors have PTEN mutations, which alter signal transduction, increase tumorigenesis, and lead to a worse patient prognosis (11). The results of the present study indicate that PTEN mRNA and protein expression levels were significantly lower in HCC compared with paracancerous tissues $(\mathrm{P}<0.05)$. These results indicated that the inactivation of the PTEN gene may play a critical role in the development of HCC. The loss of function of PTEN is mostly due to genetic changes, including point mutations, deletions, splicing alterations, frameshift mutations, missense mutations, or small fragment insertions and deletions (14). PTEN may also be posttranscriptionally regulated by miRNAs (15). We observed that PTEN expression was lower in poorly differentiated HCC with high malignancy. By contrast, it was higher in more differentiated tumors, suggesting a critical clinical significance.

miR-92 is a member of the microRNA-17-92 family and has been described to be a proto-oncogene. miR-92 is also known to promote cell proliferation and inhibit apoptosis. The present study indicates that miR-92 expression levels in HCC are significantly higher compared with those in the corresponding paracancerous tissue, which is in agreement with the study by Huang et al (16), where miR-92 was demonstrated to play a role in the development of HCC.

Despite the studies that have been performed, it remains unclear whether the regulation by miR-92 of PTEN is involved in the development of HCC. Our results indicate that the tumor suppressor gene PTEN is negatively correlated with the expression of miR-92 in HCC clinical tissues, which indicates that PTEN and miR-92 have opposing roles in HCC development which is in agreement with previous studies on other diseases (17). This result also indicates that the upregulation of miR-92 may inhibit the expression of PTEN, thereby inducing tumorigenesis through downstream genes. Consequently, the following potential mechanism of miR-92 function is proposed. The expression of miR-92 is regulated by the PI3K/ Akt signaling pathway. PTEN is a phosphatase that inhibits the PI3K/Akt pathway in normal physiological conditions, thereby inhibiting the expression of miR-92. When PTEN expression is lost, $\mathrm{PI} 3 \mathrm{~K} / \mathrm{Akt}$ is no longer inhibited, and miR-92 expression is upregulated. Cell division is promoted and the the number of cells in the $\mathrm{S}$ and $\mathrm{G} 2 / \mathrm{M}$ phases is increased. miR-92 enters the cell nucleus and binds to cell cycle-dependent protein kinases, inhibits apoptosis and promotes the proliferation of tumor cells. In the progression of HCC, miR-92 and PTEN show mutual correlation and restriction. Both miR-92 and PTEN are involved in the regulation of the cell cycle and apoptosis, whereas their biological effects are opposite $(18,19)$. In addition, it is possible that miR-92 affects Myc-induced E2F1 expression, and that it plays a role in the apoptotic response. In this case, PTEN may also play an opposing role to miR-92 $(3,20)$. Thus, examining the regulation by miR-92 of PTEN is beneficial in elucidating the complex regulatory mechanisms governing HCC tumorigenesis. Additionally, the combined detection of miR-92 and PTEN may have clinical significance in terms of diagnosis, prognostic judgment and therapeutic options for primary HCC.

\section{Acknowledgements}

This study was supported by grants from the 973 Program of China (no. 2011CB504302) and the Natural Science Foundation of Shandong (nos. 2007GG200002043 and Y2007C122). The authors appreciate valuable comments from other members of their laboratories.

\section{References}

1. El-Serag HB and Rudolph KL: Hepatocellular carcinoma: epidemiology and molecular carcinogenesis. Gastroenterology 132: 2557-2576, 2007.

2. El-Serag HB and Mason AC: Rising incidence of hepatocellular carcinoma in the United States. N Engl J Med 340: 745-750, 1999.

3. Tamguney T and Stokoe D: New insights into PTEN. J Cell Sci 120: 4071-4079, 2007

4. Martin J and Dufour JF: Tumor suppressor and hepatocellular carcinoma. World J Gastroenterol 14: 1720-1733, 2008.

5. Yao YJ, Ping XL, Zhang H, et al: PTEN/MMAC1 mutations in hepatocellular carcinomas. Oncogene 18: 3181-3185, 1999.

6. Steck PA, Pershouse MA, Jasser SA, et al: Identification of a candidate tumour suppressor gene, MMAC1, at chromosome $10 \mathrm{q} 23.3$ that is mutated in multiple advanced cancers Nat Genet 15: 356-362, 1997.

7. Lee Y, Kim M, Han J, et al: MicroRNA genes are transcribed by RNA polymerase II. EMBO J 23: 4051-4060, 2004.

8. Xie X, Lu J, Kulbokas EJ, et al: Systematic discovery of regulatory motifs in human promoters and 3' UTRs by comparison of several mammals. Nature 434: 338-345, 2005.

9. Mendell JT: miRiad roles for the miR-17-92 cluster in development and disease. Cell 133: 217-222, 2008.

10. Xiao C, Srinivasan L, Calado DP, et al: Lymphoproliferative disease and autoimmunity in mice with increased miR-17-92 expression in lymphocytes. Nat Immunol 9: 405-414, 2008.

11. Slipicevic A, Holm R, Nguyen MT, Bohler PJ, Davidson B and Florenes VA: Expression of activated Akt and PTEN in malignant melanomas: relationship with clinical outcome. Am J Clin Pathol 124: 528-536, 2005.

12. Kerr JF, Winterford CM and Harmon BV: Apoptosis. Its significance in cancer and cancer therapy. Cancer 73: 2013-2026, 1994.

13. Xu Z, Stokoe D, Kane LP and Weiss A: The inducible expression of the tumor suppressor gene PTEN promotes apoptosis and decreases cell size by inhibiting the PI3K/Akt pathway in Jurkat T cells. Cell Growth Differ 13: 285-296, 2002.

14. Smith JS and Jenkins RB: Genetic alterations in adult diffuse glioma: occurrence, significance, and prognostic implications. Front Biosci 5: D213-D231, 2000.

15. Meng F, Henson R, Wehbe-Janek H, Ghoshal K, Jacob ST and Patel T: MicroRNA-21 regulates expression of the PTEN tumor suppressor gene in human hepatocellular cancer. Gastroenterology 133: 647-658, 2007.

16. Huang YS, Dai Y, Yu XF, et al: Microarray analysis of microRNA expression in hepatocellular carcinoma and non-tumorous tissues without viral hepatitis. J Gastroenterol Hepatol 23: 87-94, 2008.

17. Huang GL,Zhang XH, Guo GL, et al: Expression of microRNA-21 in invasive ductal carcinoma of the breast and its association with phosphatase and tensin homolog deleted from chromosome expression and clinicopathologic features. Zhonghua Yi Xue Za Zhi 88: 2833-2837, 2008.

18. Fang J, Ding M, Yang L, Liu LZ and Jiang BH: PI3K/PTEN/AKT signaling regulates prostate tumor angiogenesis. Cell Signal 19: 2487-2497, 2007.

19. Mann CD, Neal CP, Garcea G, Manson MM, Dennison AR and Berry DP: Prognostic molecular markers in hepatocellular carcinoma: a systematic review. Eur J Cancer 43: 979-992, 2007.

20. O'Donnell KA, Wentzel EA, Zeller KI, Dang CV and Mendell JT: c-Myc-regulated microRNAs modulate E2F1 expression. Nature 435: 839-843, 2005. 